\title{
Degradation of non-methylated DNA by MsPJI impairs its usefulness as epigenetic tool
}

María Belén Jerez* and Maximiliano Juri Ayub

\section{Affiliation:}

Instituto Multidisciplinario de Investigaciones Biológicas de San Luis, IMIBIO-SL-CONICET and Facultad de Química, Bioquímica y Farmacia, Universidad Nacional de San Luis, San Luis Argentina.

*Corresponding Author. E-mail: jerezbel@gmail.com

\begin{abstract}
DNA treatment with sensitive and/or dependent restriction enzymes, followed by PCR amplification is a widely used approach for testing CpG methylation. Recently, MspJl has been characterized as a promisory tool for epigenetic analyses. In the present report, we describe that MspJl shows significant activity against non-methylated DNA, suggesting that additional caution and improvements would be required before applying this enzyme as a routine epigenetic tool.
\end{abstract}

\section{INTRODUCTION}

In the last years, research on the epigenetic DNA modification has increased exponentially. In particular, knowing the methylation state of cytosine residues at CpG sites in animal genomes is a widely addressed issue, because 5-methylcytosine has been implied as a major regulatior of gene expression [1].

Two general basic approacches have been used for detecting cytosine methylation; namely bisulfite conversion and restriction with methylation-sensitive or dependent enzymes. The bisulfite based methods are powerful but cumbersome, since bisulfite 
modified DNA is unstable, containing many strand breaks, and difficult to amplify. Therefore, restriction based approcches are of choice for non-specialized laboratories. One of these approaches is based on the combination of genomic DNA restriction with PCR (preferably qPCR) amplification, using primers flanking the DNA region under study [2] (Figure 1). Recently, a novel and promisory methyl-dependent enzyme has been described: MspJI [3]. This enzyme has been characterized and described as cleaving the mCNNR sequence, being inactive against non-methylated sequences. For this reason, it has been postulated as a powerful tool for epinegetic studies, being able to detect around $50 \%$ of methylated cytosines at CpG sites. Therefore, we tested this enzyme as a tool for the specific detection of cytosine methylation on mammalian genomic DNA.

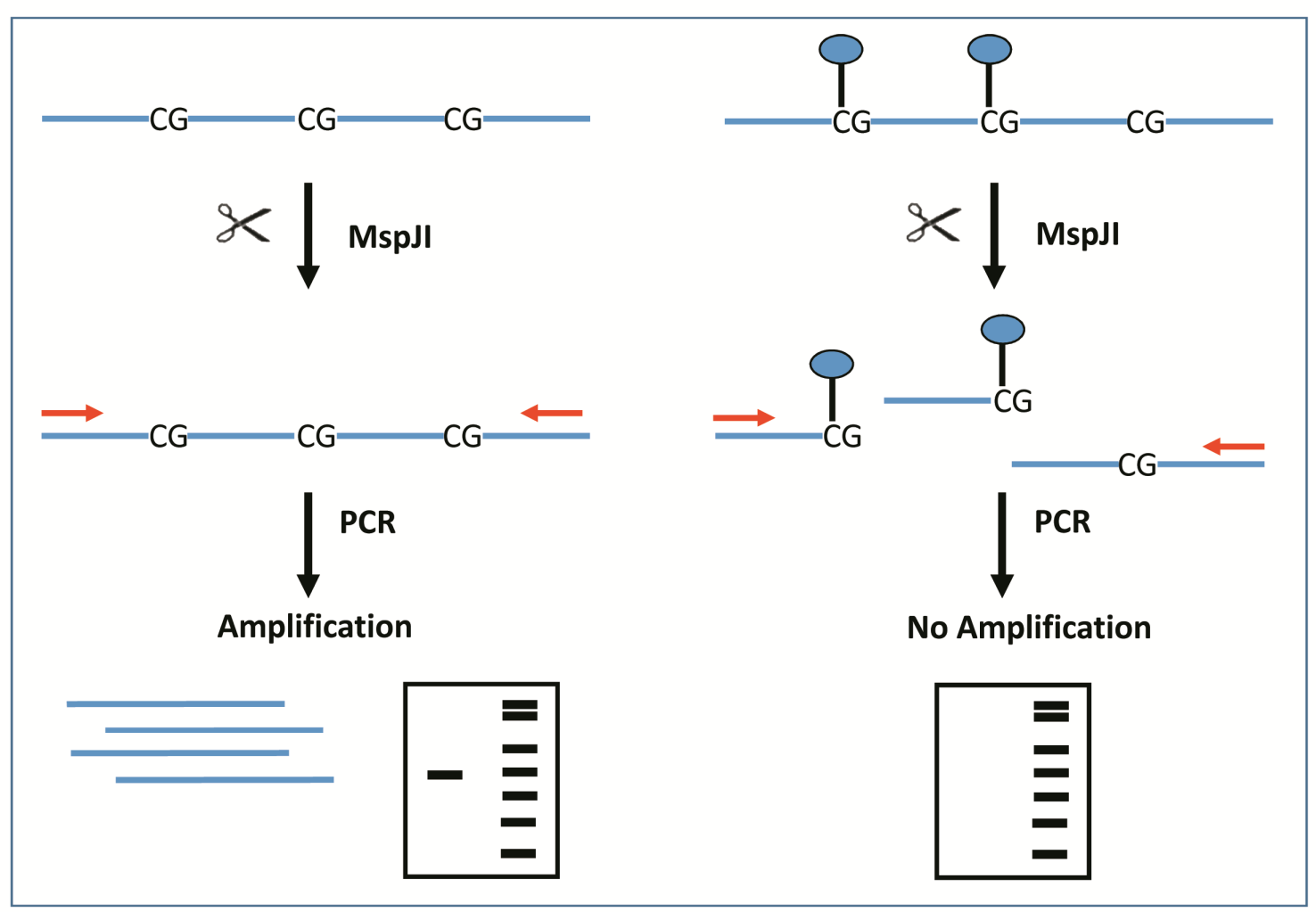

Figure 1. Schematic representation of the experimental design. Genomic DNA is treated with MspJI. If DNA molecules are non-methylated (left), template remains intact and can be amplified by PCR. If DNA is methylated (right), MspJl degrades template and weaker or no amplification take place. Partial methylation can be better detected by qPCR as a shift in Cq values. 


\section{METHODS}

\section{DNA purification}

DNA was purified from mice peritoneal macrophages or G. lamblia axenic cultures using the Wizard® Genomic DNA Purification Kit (Promega), following the manufacturer instructions. Integrity of DNA was confirmed by agarose electrophoresis and quantification was carried out by absorbance at $260 \mathrm{~nm}$ using spectrophotometer (BioTek Instruments, Epoch ${ }^{\text {TM }} /$ Take3 $^{\text {TM }}$ Multi-Volume Plate).

\section{Digestion with MspJI}

Genomic DNA $(1 \mu \mathrm{g})$ was digested following the manufacturer instructions, using 2 unit of MspJI (New England Biolabs) in the presence of $1 \mu$ double-stranded DNA activator in a $30-\mu \mathrm{L}$ volume. All reactions were incubated at $37^{\circ} \mathrm{C}$ for $4 \mathrm{~h}$ or $8 \mathrm{~h}$. The digestion products were visualized on $1 \%$ agarose electrophoresis.

\section{End-point PCR}

PCR reactions were performed using $50 \mathrm{ng}$ of genomics DNA incubated at $37^{\circ} \mathrm{C}$ in the presence or in the absence of MspJl. The following oligonucleotides were designed in order to amplify a 1,799 bp fragment from the IL-12b FW: 5' TCGGCCCCATATTGCTTTGT 3' and Rev: 5' ACAGCCTCTAGATGCAGGGA 3'. Thermocycling conditions were as follows; 1 cycle at $94^{\circ} \mathrm{C}$ for $30 \mathrm{sec} ; 35$ cycles of $94^{\circ} \mathrm{C}$ for $30 \mathrm{sec}, 63^{\circ} \mathrm{C}$ for $45 \mathrm{sec}, 72^{\circ} \mathrm{C}$ for 2 min ; followed by a final elongation step at $72^{\circ} \mathrm{C}$ for $10 \mathrm{~min}$. Amplicons were visualized by electrophoresis on $1.5 \%$ agarose gel.

\section{Real-time PCR}

PCR amplification mixtures contained $50 \mathrm{ng}$ of genomic DNA, FastStart Universal SYBR Green Master (Rox) (Lifescience. Roche), 500 nM primers and UltraPure ${ }^{\mathrm{TM}}$ DNase/RNaseFree Distilled Water (Life technologies). Reactions were run on an ABI PRISM 7500 (Applied Biosystems). The cycling conditions comprised $2 \mathrm{~min}$ at $50^{\circ} \mathrm{C}, 10 \mathrm{~min}$ polymerase activation at $95^{\circ} \mathrm{C}$ and 40 cycles at $95^{\circ} \mathrm{C}$ for $15 \mathrm{sec}$ and $60^{\circ} \mathrm{C}$ for $60 \mathrm{sec}$. The following primers were designed to amplify a 95bp CpG fragment of the beta actin gene : Fw 
CtTGAtCTTCATGGTGCTAGgAg and Rev CAGTGCTGTCTGGTGGTAC. For PCR amplication of the $333 \mathrm{bp}$ fragment from the IL-12b promoter, the following primers were used FW: AAG TGT GTG GCT GGG AAG and Rev: GTT GAT GTT ACC TCCC TTCCTC.

\section{RESULTS AND DISCUSSION}

Figure 2 shows the digestion pattern after incubation of murine genomic DNA with MspJl. As expected, a smeared pattern was observed, consistent with the digestion of a densely methylated DNA. On the other hand, when genomic DNA of the protozoan Giardia lamblia showed a pattern similar to undigested sample. These results are consistent with a highly specific digestion of methyled DNA by MspJl, according to previous reports.

\section{M. musculus DNA}

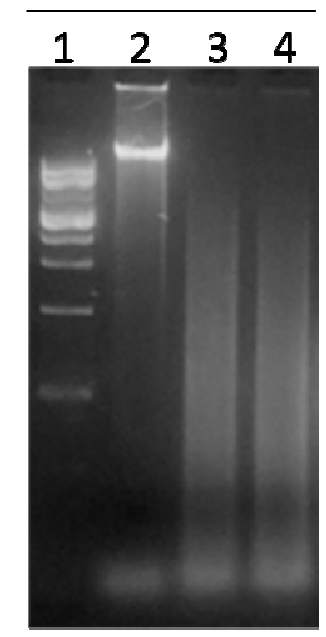

\section{G. lamblia DNA}

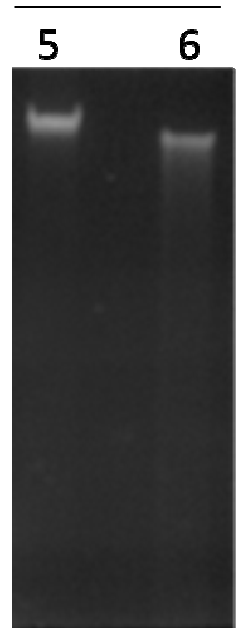

Figure 2. Electrophoresis of genomic DNA from M. musculus or G. lamblia digested with MspJl. Lane 1: $1 \mathrm{~kb}$ DNA ladder; lane 2 and 5: undigested DNA; lanes 3 and 6: DNA incubated with MspJl for 4 hs; lane 4: DNA incubated with MspJl for 8 hs.

Next, we used MspJl digested mice DNA as template to amplify a 1,799 bp fragment from the IL-12b gene (GenBank: AH004859) (nt -1693bp to +106 bp, numbers according to the transcription initiation site), containing 39 CpG sites. Notably, digestion with MspJl completely prevented the amplification of the selected region, suggesting a high methylation state (Figure $\mathbf{3}$ ). 


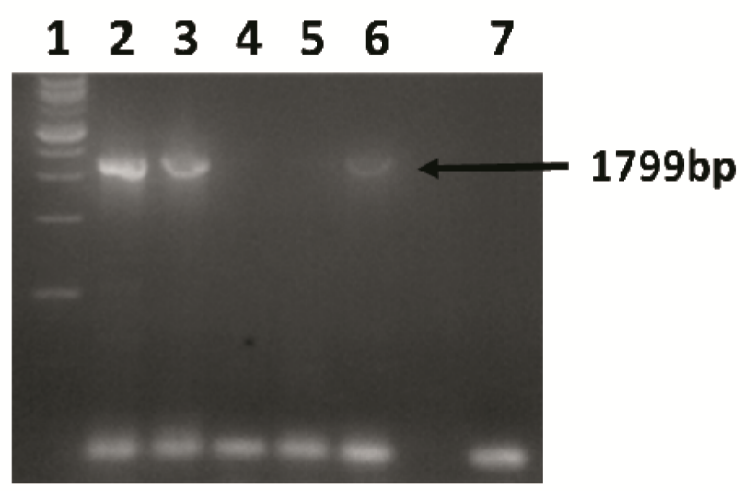

Figure 3. PCR amplification of IL-12b promoter gene. Genomic DNA was incubated in the absence (lanes 2 and 3) or the presence (lanes 4 and 5) of MspJl, and used as template. Positive (lane 6) and negative (lane 7) amplification controls were analyzed in parallel.

This result prompted us to set a quantitative amplification analysis by using QPCR instead of end-point PCR. We selected two different PCR targets: a -333 bp sub fragment on the IL-12b promoter including 14 CPG sites (a CPG island; nt -1136 to -801, numbers according to the transcription initiation site) and a $95 \mathrm{bp}$ fragment belonging to betaactin gene (GenBank: NC_000071.6; nt 142904067 to 142904161, nt +999 to +1093 according to the transcription initiation site) lacking of CPG sites. Surprisingly, in both cases, enzyme treatment produced a 2-3 cycle's shift of the Cq value (Figure 4), showing that DNA degradation was not dependent on CpG methylation.

Although this would be rather unusual, it can be postulated that methylated cytosines at sequences other than CPG were present in the genomic samples and recognized by MspJI. In fact, it has been reported non-CpG methylation in stem cells and, more recently, in differentiated mammalian cells [4]. Therefore, in order to evaluate the specificity of the assay using a bona fide non-methylated sample, we replaced genomic DNA template by a diluted PCR product. Notably, treatment of this non-methylated DNA sample with MspJI, PCR amplification was prevented, definitively confirming that MspJI degrades non-methylated DNA at a significant extension. These results demonstrate that MspJl is inadequate for epigenetic analyses based on methylation-dependent restriction coupled to qPCR strategy (Figure 5). 


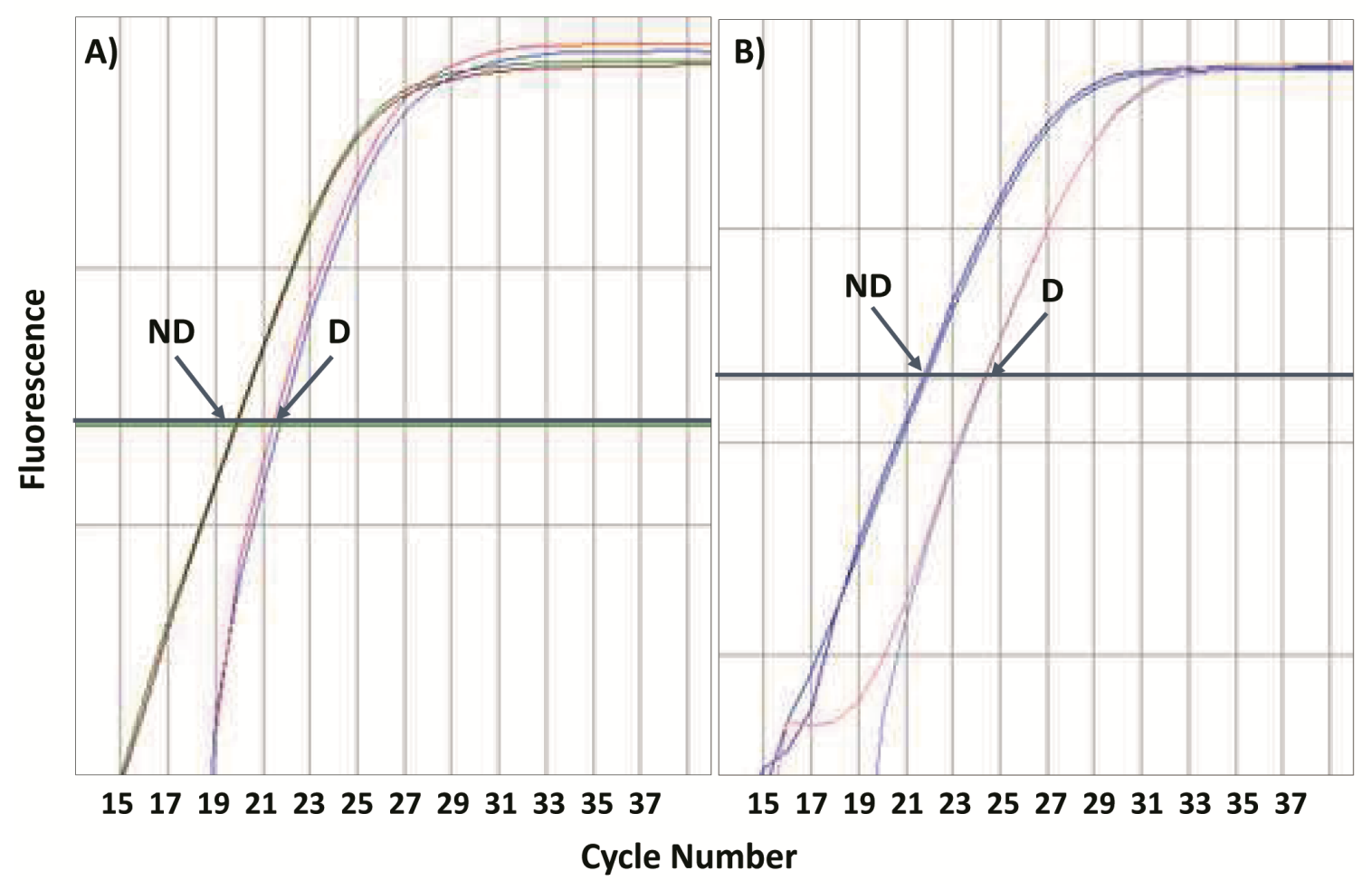

Figure 4. Effect of MspJI treatment on the real time amplification of genomic templates. qPCR reactions were performed using murine genomic DNA digested (D) or not digested (ND) with MspJl. The target sequences contained 14 (A) or none (B) CPG sites. The curves are representative of 12 independent experiments performed in duplicates.

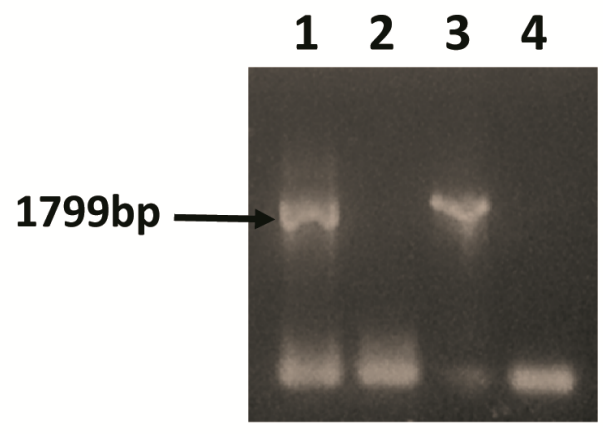

Figure 5. Effect of MspJI on the amplification of non-methylated DNA. PCR product of 1799 bp was incubated in the absence (lane 1) or presence (lane 2) of MspJI. Treated samples were re-amplifiied by PCR. Positive (lane 3) and negative (lane 4) controls were included. 
Concluding, we have found that even when MspJl selectively degrade methylated DNA, its activity against non-methylated DNA is not negligible. Therefore, cautions should be taken when using this enzyme for epigenetic studies.

\section{ACKNOWLEDGMENTS}

We are grateful to Dr. Maria L. Mascotti and Dr. Walter J. Lapadula for their helpful comments and criticisms about the manuscript.

\section{REFERENCES}

1. Deaton AM, Bird A (2011) CPG islands and the regulation of transcription. Genes Dev 25: 1010-1022.

2. Hashimoto K, Kokubun S, Itoi E, Roach HI (2007) Improved quantification of DNA methylation using methylation-sensitive restriction enzymes and real-time PCR. Epigenetics 2: 86-91.

3. Cohen-Karni D, Xu D, Apone L, Fomenkov A, Sun Z, et al. (2011) The MspJl family of modification-dependent restriction endonucleases for epigenetic studies. Proc Natl Acad SCi U S A 108: $11040-11045$.

4. Pinney SE (2014) Mammalian Non-CpG Methylation: Stem Cells and Beyond. Biology (Basel) 3: 739-751. 